\title{
Inquiry Learning Methodologies and the Disposition to Energy Systems Problem Solving
}

\author{
Minha R. Ha \\ York University \\ $\underline{\text { minhareo@yorku.ca }}$ \\ Shinya Nagasaki \\ McMaster University \\ nagasas@mcmaster.ca \\ Justin Riddoch \\ McMaster University
}

\begin{abstract}
In this paper, we argue that it is essential to pay attention to the engineering students' use of sound methodologies in approaching engineering problems. There are serious challenges created from surface learning attitudes that undermine foundational, conceptual understanding and basic methods to solve technical problems. Moreover, such attitudes carry over to how students approach the complexity and human aspect of engineering problems. Senior undergraduate energy systems courses were redesigned to develop students' inquiry and problem solving skills. Data from a post-course survey, completed by 58 senior engineering students, were analyzed using a thematic analysis and basic categorization. Findings suggest that inquiry learning (IL) and problem based learning (PBL) methods offer much value in the students' development of research and analytic skills. As well, students gained a deeper appreciation of complexity and the ethical issues in energy system challenges, which may have some impact on their assumed responsibility as engineers - during the process and in the aimed outcomes of their problem solving tasks. We reflect on the findings to propose how $I L$ and PBL might be effectively designed and implemented for engineering students engaged in systemlevel analyses.
\end{abstract}

Keywords: Problem-based learning; Inquiry learning; Energy systems; Learning methodologies

\section{INTRODUCTION}

The complex and interconnected nature of the engineering industry is placing a greater demand on the universities to bring multi-disciplinary education to undergraduate students. Greater emphasis is being placed on systems-level problem solving, which involves intersecting systems of technology, economy, governance, culture, geography, and many others [3]. Considering the engineering programs within Ontario alone, complementary studies are being offered through courses in other Faculties, or in special courses where instructors from multiple disciplines collaborate. However, the challenge of integrating multidisciplinary content and skills in engineering problems still remains. Such learner abilities are difficult to assess or teach, for engineering instructors who have not been trained to do so.

At McMaster, two senior undergraduate courses (EP 3ES3 and EP 4ES3) are offered on the topic of energy systems (mainly enrolling students from the Departments of Civil Engineering and Engineering Physics). These courses address not only the conventional engineering (e.g. physics, chemistry, thermodynamics, materials) but also the interface between the technologies and human beings and society (i.e. ethics, stakeholders). System-level considerations, where one sees interacting parts of the whole, inherently require accurate understanding of the needs and conditions of the stakeholders. Students need training and resources to define energy problems beyond the technological aspects, and to develop effective strategies towards offering solutions.

Because of previous successes of problem-based learning (PBL) in engineering and health sciences at McMaster, we asked whether PBL principles and frameworks could be adopted to help develop multidisciplinary perspectives in approaching energy system problems. At the same time, we recognized that undergraduate students may need a systematic training on the process of investigation, much like research skills where problems are open-ended and the investigator needs to gather and analyze data as appropriate to the 
nature of the studied phenomena. Building on the existing inquiry project format in EP 4ES3, we asked whether a structured inquiry report, which makes explicit necessary components and evaluation criteria, would help train students for methodical problem solving. Both PBL and inquiry learning (IL) emphasize the learners' process in solving open-ended, system-level engineering system problems; it is in this process that we aimed to integrate multi-disciplinary perspectives.

In order to evaluate the effectiveness of the PBL and IL methods, pre-course and post-course surveys were conducted. Open-ended and rating responses were collected from both EP 3ES3 and EP 4ES3 students. This paper draws from the EP $3 \mathrm{ES} 3$ post-course survey data, which include student responses to rating questions and open-ended questions. 58 students (of 64 students enrolled in EP 3ES3) participated. A thematic analysis was conducted on the open-ended responses, which helped answer the following questions: How effective were the PBL and IL approaches? Which learning outcomes were most affected, and why? What are the factors that contribute to successful learning, for the target learning outcomes?

\section{COURSE DESIGN}

The third-year energy systems course was redesigned to target the development of systems thinking, and rigorous inquiry skills. Two PBL tutorials (one week each) were designed, heavily involving group work activities. A mini-inquiry project (seven weeks) was introduced for the first time, which aimed to prepare students for their major inquiry project in the fourth year energy systems course. The existing course content (e.g. analytic models and drill exercises) were delivered through conventional lectures and remaining tutorials. All the course content, including PBL/IL materials, were provided in two course handbooks.

It is important to provide guidance in new learning methods, to scaffold learning and to reduce cognitive load [2]. Thus, we first structured the mini-inquiry project into smaller components (e.g. problem statement, methodology) to be submitted by scheduled deadlines throughout the term. A workshop with the librarians on campus trained students on scholarly literature search and citation practices, and the teaching assistants provided feedback on the submitted inquiry components. We also introduced existing problem solving method frameworks [4]. in tutorials, to broaden the way students would explore, define, and analyze open-ended problems.

\section{EFFECTIVENESS OF PBL AND IL}

\subsection{Most Effective Teaching Methods}

CEEA16; Paper 086

Dalhousie University; June 19 - 22, 2016
Students were asked to identify which component of the course helped them learn their top 'take-aways.' In this way, the most valuable instructional components of the course were identified as (of 49 responses): (a) the group work process [during IL/PBL activities] (35\%); (b) the inquiry report $(20 \%)$; (c) the PBL assignments (18\%). Other responses included: (d) lectures and drills (14\%); and (e) individual research (8\%). Course handbooks, teaching assistants, and research skills session were each mentioned once.

\subsection{Effective Features of PBL and IL}

Students were asked to comment on the effectiveness of PBL and IL implementation in the course. For PBL, 26 students identified its effective features, and 19 students identified areas for improvement; For IL, 28 students identified effective features, and 17 described barriers to effectiveness. Results for PBL and IL were combined, to develop categories of features that accommodate both methods. Each category is followed by student quotes from the survey.

Overall, the IL/PBL methods worked for some students because:

a) Focus on skills development: Research, presentation, group work

"[PBLs] were also very effective specially in toning and enhancing professional writing skills. " [Student 3]

"PBLs were effective at promoting independent group work and brainstorming. " [Student 28]

"[IL] improve student's researching skill a lot. and also the improve student's data analysis skill. and also it improve student's communication skill." [Student 50]

"Very effective at supplying an answer to a question with an extreme amount of proof with it." [Student 56]

\section{b) Connection to real world problems}

"The PBL exercises offered a good opportunity to apply the concepts learned in the course to real world situations. I thought both PBL's were good real world issues and using groups was an effective way to share ideas and information." [Student 48]

"The IL in this course were probably the most effective at enhancing the learning experience of the students. It forced us to investigate and ask questions about real world problems and to think about how these problems could ACTUALLY be solved. The group work was practical and realistic to real engineering jobs and made it a learning experience over all." [Student 54]

\section{c) Structured process for PBL and IL}

"Effective. PBL 1 was well defined and therefore our group learned a lot from the exercise. PBL 2 was poorly explained and broad, which made it less applicable." [Student 13] 
"[IL] was decent. Weekly submissions kept us on track, and it demonstrated an effective process to approach inquiry based learning." [Student 13]

"The IL was effective at showing how to break down a problem to make it easier to solve." [Student 45]

\section{d) Clear connection between course activities}

"I think the PBL exercises were also very effective. They helped to develop the research skills that were necessary for the IL tasks. It was very structured as to what the problem statement was so it was easy to get started and come up with a solution. It also encouraged us to determine quality resources that are reliable so we could draw realistic conclusions." [Student 19]

\subsection{Barriers to PBL and IL Effectiveness}

The IL/PBL methods did not work for some students because:

\section{a) Lack of clear guidance or quality feedback from instructional staff}

"The IL in this course was an interesting project to handle. Due to the lack of guidance, starting off the $I L$ was difficult but as progress was made and the scope and questions were refined, it became easier and more beneficial to complete. Overall, it was somewhat effective." [Student 19]

"I found there wasn't enough feedback on inquiry 1 and 2. I think there should have been more discussion around the creating the problem statement and how to develop a suitable question. I found our group had no direction and when it came to the presentation we were told to present a solution to the problem. We did not realize that we had to come up with a solution to the problem, we were more focused on finding out why and explaining why the problem existed." [Student 18]

"The IL could have been done better by giving a better class on choosing a good topic for the IL. The IL could have had more pointed questions during the IL checkpoints to cause a bit more research to be done throughout the weeks rather than all at once near the end. " [Student 45]

\section{b) Unfamiliarity with the topic of energy systems and open-ended problems \\ "Minimal guidance and poor structure; too open ended.} Our written report was very vague as we don't have the extensive background in the field for which research was to be conducted." [Student 33]

"[IL] relied much too heavily on people already being invested in having a topic and each 'checkpoint' served as another deadline to meet rather than a step to take to get closer to an answer. Any information you obtained was by your own choice and searching. This is alright for one specific topic but you get nothing out of the 'big picture'." [Student 40]

\section{c) Limitations in fundamental knowledge}

Because the success in EP $3 E S 3$ also required technical competencies, students' varied levels of competency with the fundamental concepts also became a factor to their success in mini-inquiry project. Unexpectedly we found that basic mathematical skills or fundamental knowledge from sciences were lacking, and creating a limitation to students' success in inquiry project. This includes the students' ability to translate between qualitative phenomena and quantitative values, in basic physics, chemistry and mathematics. For example, in analyzing climate change, the first step is to consider the balance between the energy from the sun and the energy released from the earth, and the energy fluxes can be discussed based on the basic physics such as black body radiation (flux $=T^{A}: \quad$ is a physical constant and $T$ is absolute temperature) and conductive heat transfer (flux $=C\left(T_{\text {high }}-T_{\text {low }}\right)$ in simple case, where $C$ is a constant depending on materials). In economic analysis, for example, future value $=$ present value $\times(1+r)^{n}$, where $r$ is interest and $n$ is a number of years of project. This is a simple sum of geometric progression which is taught in high school. Although the economic analysis was studied through exercises, quiz and assignment, many students of both departments struggled with these equations in their inquiry and their final exam.

\section{IMPACT OF PBL AND IL ON STUDENT LEARNING}

\subsection{Key Learning Outcomes}

Students were asked to identify their top three take-aways from the $3 \mathrm{ES} 3$ course. Three key categories of learning outcomes emerged (122 items total):

i. Methodologies in solving energy systems problems (50\%), including:

research and writing skills, data analysis and interpretation (e.g. quantitative and qualitative), professional communication, group work, evaluation of scholarly literature, problem analysis

ii. A deeper understanding of energy systems (25\%), including:

their impact, variation, complexity (e.g. societytechnology relations, variables), and criteria for evaluation (e.g. sustainability, accessibility); and,

iii. Awareness of the current issues and trends, both local and global (20\%).

\subsection{Profiles of Student Approaches to Open-Ended Questions}

In the same exit survey, two open-ended questions were asked that offered insight into the categories (i), (ii) and (iii) above. These questions reflect two of the important themes that emerged from student work during the course: 
the decision making process involved in energy system solutions, and the potential for sustainability and equity in the Canadian energy systems. The students' written responses were profiled based on their similarities.

When asked to outline the decision-making process that they would undertake in order to design a new energy systems strategy in Ontario:

- 15 students had no conceptual framework for the decision making process (Profile A);

- 13 students stated the importance of proper decision making, but described no specific model (Profile B);

- 10 students resorted to the cost-benefit analysis as the sole decision making tool (Profile C); and,

- 9 students provided the steps of the decision making process, but did not identify which stakeholders are responsible or have the authority to conduct each action - especially the final steps (Profile D).

Overall, students showed a wide variance in their ability to develop a systematic procedure to approaching a complex problem.

When asked to identify opportunities in advancing energy systems in the Canadian context:

- 16 students made no identification of any strengths or opportunities in Canada for energy systems;

- 13 students asserted that engineers should dictate the discussion or direction of energy system development in Canada;

- 8 students discussed the potential strength of Canadian diversity in technological development within Canada, and Canada's role in the international community;

- 13 students highlighted the potential role of strong, transparent regulations in advancing energy systems;

- 4 students stated that the combination of regulation, engineering profession, diversity and others would induce the good interactions between the technology and the society and establish the informed consensus in the society; and,

- 6 students emphasized the ethical issues that must be addressed in relation to the strengths and opportunities in Canada, including engineers' responsibility.

Overall, students demonstrated a moderate level of conceptual understanding in terms of the inter-related systems surrounding energy issues. There was generally a weak reference to actual trends and debates in our local contexts.

\section{DISCUSSION}

\subsection{Importance of Process Skills}

Multi-disciplinary education suggests that the learners are able to apply qualitative and quantitative methods appropriate to studying different kinds of phenomena. In reviewing the learning outcomes of the course from students' and instructors' perspectives, it is quite concerning that students lack meaningful application of basic scientific equations, let alone the ability to conduct analyses of social scientific data. Many students solve well-defined problems in their engineering courses, and later engage in capstone projects. In developing systemlevel analytic skills in students, however, more rigorous training for investigating multi-disciplinary phenomena may be required throughout the undergraduate program.

There was moderate success in developing process skills in EP 3ES3 students, namely research and communication (i.e. writing, group work, data presentation) skills. The discipline in proper inquiry skills (i.e. problem statement development, research question design, appropriate methodology selection, evaluation of data sources, data analysis and interpretation) should not be compromised in the university-level education. It is expected that the careful approach to problem solving will translate from one context to another.

Students showed weak to moderate ability to develop their own procedures in approaching an open-ended problem. Before students are assigned with independent projects, it would be greatly beneficial to help students learn to integrate various analytic models, problem solving methods, and decision making frameworks. Learning habits should be examined as well [1].

\subsection{Implementation of PBL and IL}

Three important factors emerged as contributing to the varied experience with PBL and IL implementation:

- Instructional staff levels of skills in facilitation, feedback, and guidance - The teaching assistants (TAs) had different levels of familiarity with the current energy systems issues and methodologies. The TAs, course instructor and other educational staff also had different levels of training in class facilitation, instructional feedback, and guidance in PBL and IL. Students desired more instructional input in successfully completing PBL and IL activities.

- $\quad$ Structure of course design, as well as course content - Much of the successes in the IL implementation came from the course structure. Manageable deliverables and useful educational resources (i.e. Course handbook) were important in helping students succeed in the course.

- Student level of research skills and experience with open-ended problems, motivation to engage in independent learning - Civil engineering students had more exposure to PBL approaches from previous 
courses than engineering physics students. Students had varied work and research experiences, which may have contributed to different levels of comfort with PBL and IL activities.

Two of the three factors stress the importance of developing instructional staff for effective pedagogy. Pedagogical development thus creates an opportunity for developing multi-disciplinary competencies in the engineering graduate students and faculty. Partnership with the university teaching and learning centre, as well as with instructors across Faculties, may provide excellent opportunities for developing the instructional staff.

Program-level discussions may also be important. Course design takes into account student workload, to adequately organize assessment deliverables. Learning experiences and outcomes from prior courses also orient students for particular ways of learning. For effective system-level inquiry projects in senior years, problem solving and research skills may need to be introduced in earlier years of the program.

Learning is a collaborative endeavour between students and the instructional staff. Educators must make an effort to discuss the aims and logic of their pedagogy, and invite students to help maximize the value of learning strategies such as PBL and IL. Overlooked opportunities to discuss expectations and learner needs may delay student progress.

\section{CONCLUSION}

Well-structured PBL and IL strategies offer much potential in developing key process skills in engineering students. Developing a systematic framework for solving system-level problems involve an integration of analytic methods and knowledge from multiple disciplines; PBL and IL strategies may create opportunities for students to exercise and develop such skills. This kind of pedagogical development, however, must include developing instructional skills in faculty and teaching assistants, as well as program-wide collaboration to develop effective learner habits and approaches to engineering problem solving.

\section{Acknowledgements}

This study and course redesign work were made possible by the Teaching and Learning Grant from McMaster Institute for Innovation and Excellence in Teaching and Learning.

\section{References}

[1] Biggs, J. (1999). Teaching for enhanced learning. http://doi.org/0729-4360/99/010057-19

[2] Hmelo-Silver, C. E., Duncan, R. G., \& Chinn, C. A. (2007). Scaffolding and achievement in problem-based and inquiry learning: A response to Kirschner, Sweller, and Clark (2006). Educational Psychologist, 42(2), 99107. http://doi.org/10.1080/00461520701263368

[3] Senge, P. (1994). The Fifth discipline fieldbook: strategies and tools for building a learning organization. New York: Currency Doubleday.

[4] Woods, D. R. (1998). the MPS program: the McMaster Problem Solving program. McMaster. Retrieved from http://chemeng.mcmaster.ca/sites/default/files/media/mps 2.pdf 